\title{
LOS SUPUESTOS BÁSICOS SUBYACENTES DE LOS DOCENTES DE CIENCIAS VETERINARIAS DE LA UNIVERSIDAD NACIONAL DE ROSARIO
}

\author{
Rodolfo Darío García* \\ Universidad Nacional de Rosario, Argentina \\ rodolfodgarcia@gmail.com \\ Griselda Muñoz** \\ Universidad Nacional de Rosario, Argentina \\ mgriselda01@gmail.com
}

Recibido: 25/07/2019 Aceptado: 3/11/2019

\section{Resumen}

La labor de los docentes universitarios se desarrolla de múltiples maneras. Ellos eligen sus estrategias didácticas a partir de lo que Alvin Gouldner llamó Supuestos Básicos Subyacentes (SBS). Este estudio tuvo el objetivo de reconocer y comprender algunos posibles SBS de los docentes de la carrera de Medicina Veterinaria de la Facultad de Ciencias Veterinarias de la Universidad Nacional de Rosario (UNR). Se trata de una indagación de tipo empírica, cualitativa y transversal realizada durante el año 2017. Los SBS seleccionados fueron las concepciones acerca de la realidad, la ciencia, el conocimiento y el aprendizaje. Se realizaron entrevistas en profundidad a los profesores a cargo de las cátedras del ciclo básico. Para el procesamiento de los datos y la construcción de las categorías de análisis, se utilizó el programa Atlas Ti 8.4 ${ }^{\circledR}$. Los principales hallazgos demuestran la existencia de SBS mayormente ligados a una concepción positivista/conductista que a una concepción constructivista.

Palabras clave: Supuestos básicos subyacentes - Docentes - Ciencias veterinarias - Conductismo - Constructivismo.

\section{Abstract}

The work of university teachers develops in multiple ways. They choose their didactic strategies from what Alvin Gouldner called Background Assumptions (SBS). This study aimed to recognize and understand some possible SBS of teachers of the Veterinary Medicine career of the Faculty of Veterinary Sciences of the National University of Rosario (UNR). It is an empirical, qualitative and transversal inquiry carried out during the year 2017. The SBS selected were the conceptions about reality, science, knowledge and learning. In-depth interviews were conducted with the professors in charge of the basic cycle chairs. For the processing of the data and the construction of the analysis categories, the Atlas $\mathrm{Ti} 8.4 \AA$ program was used. The main findings show the existence of SBS mainly linked to a positivist / behavioral conception that to a constructivist conception.

\footnotetext{
*Alumno de la carrera Maestría en Educación Universitaria de la Facultad de Humanidades y Artes de la UNR (Tesis en evaluación). Médico Veterinario en ejercicio de la profesión. JTP de la cátedra Anatomía Descriptiva y Comparada II de la Facultad de Ciencias Veterinarias de la Universidad Nacional de Rosario. Docente de la Especialización en Medicina de Felinos Domésticos.

**Magister en Educación Universitaria y Profesora en Veterinarias por la Facultad de Humanidades y Artes de la UNR. Médica Veterinaria. Vicedecana y Profesora Adjunta de la Facultad de Ciencias Agrarias de la UNR. Docente investigadora Categoría II. Directora de proyectos de investigación sobre problemáticas educativas en la universidad. Directora de tesis en la Maestría en Educación Universitaria de la Facultad de Humanidades y Artes de la UNR. Docente de la Maestría en Docencia en el Campo de la Formación en Prácticas Profesionales de la Facultad de Humanidades y Artes de la UNR.
} 
Keywords: Background assumptions - Teachers - Veterinary Sciences - Behaviorism, Constructivismo.

\section{Introducción}

Los docentes universitarios desarrollan sus tareas de enseñanza de maneras muy diversas. Utilizan estrategias didácticas variadas que van desde la tradicional exposición magistral hasta el desarrollo de propuestas en las que los estudiantes participan activamente a través del debate, la elaboración de trabajos colaborativos, la resolución de problemas o el estudio de casos. Como es sabido, los docentes eligen sus estrategias didácticas guiados por ciertas concepciones, ciertos supuestos, denominados según la construcción teórica propuesta por Alvin Gouldner (1970) Supuestos Básicos Subyacentes (SBS). Según este autor, los SBS son "herramientas cognitivas cargadas de afectividad que surgen en los comienzos de nuestra socialización dentro de una cultura particular y se hallan profundamente arraigadas en nuestra estructura de carácter" (Gouldner, 1970, s/p). El concepto de SBS se refiere a las concepciones que cada uno produjo acerca de qué son el Hombre, el Mundo, la Educación y las relaciones sociales. Se originan a partir de sus experiencias, sus prejuicios, sus creencias, su identificación con cierto estrato social, disciplina o comunidad científica y sus lealtades políticas e ideológicas, entre tantas otras.

Son supuestos porque en general no son factibles de ser demostrados o no hay preocupación porque así sea. Son básicos porque están en el origen más profundo y a veces oculto de las teorías y las prácticas. Son subyacentes porque no se hacen explícitos, a veces ni para quien los sostiene (Sanjurjo, 2003, p. 20).

La gama de SBS es muy amplia: hay algunos muy globales, como la concepción de que es el Hombre o el Mundo, y otros más restringidos que se derivan de los primeros. En este trabajo en particular, nos enfocaremos en las concepciones acerca de la realidad, la ciencia y el conocimiento; y en las concepciones acerca del aprendizaje. Consideramos que estos son algunos de los principales SBS que orientan al profesor en la selección de las estrategias didácticas que conformarán su propuesta de enseñanza.

Destacamos que este estudio forma parte de una investigación más amplia, enmarcada en una tesis de maestría cuyo objetivo fue comprender la relación entre los SBS y las estrategias didácticas que utilizan los profesores del ciclo básico de la carrera Medicina Veterinaria.

\section{Las concepciones acerca de la realidad, la ciencia y el conocimiento}

A lo largo de la historia la Humanidad ha forjado numerosas y muy variadas formas de conceptualizar el conocimiento y la realidad. También concibió otras tantas maneras en las que las personas pueden acceder a ellos. Como su enumeración, desarrollo y explicación exceden los objetivos de este trabajo sólo se considerarán las dos corrientes de pensamiento que tuvieron mayor influencia en la forma de entender el conocimiento durante el siglo XX y lo que va del siglo XXI.

Una de ellas, el positivismo, hunde sus raíces en la corriente filosófica conocida como realismo ingenuo. Ella parte de la premisa de que la realidad es algo exterior a la persona y que tiene existencia por sí misma. Considera que su inteligibilidad no depende de la consciencia de la persona humana (Daros, W. 1983, p. 89). Heredero del pensamiento iluminista, el Positivismo se consolidó como el paradigma hegemónico en cada una de las actividades sociales, incluidas la ciencia y la educación, desde finales del siglo XIX hasta nuestros días.

El positivismo pedagógico acompañó el surgimiento de los países europeos entre los siglos XVIII y XIX. Se transformó en una herramienta de cambio social en los países americanos hacia fines del siglo XIX, incluso en la República Argentina. Efectivamente, esta corriente de pensamiento, cimentada en el concepto de que el conocimiento es algo dado, uniforme, único y adquirible por el sujeto, armonizaba con las necesidades de un Estado argentino que a mediados del siglo XIX estaba en formación. Las ideas de orden, progreso indefinido y pensamiento único sustentaban la ilusión de pacificar al país, que por aquel entonces se encontraba inmerso en luchas internas. Se consideraba que si las clases sociales más relegadas y la población indígena conocían la realidad podrían salir del oscurantismo en el que estaban subsumidos y sumarse a la cultura y a la sociedad. Se pretendía por esta vía lograr la unidad nacional (Entel, 1988, pp. 10-11). El sistema educativo argentino se estructuró bajo estas premisas. Aunque esta forma de pensamiento comenzó a ser criticada hacia mediados del siglo XX y en la actualidad se considera superada, al menos en forma inconsciente, sigue teniendo una influencia decisiva en el pensamiento y las concepciones de aquéllos que participan del intercambio educativo. Esta influencia se hace patente en los documentos oficiales, los proyectos curriculares, los textos elaborados y las estrategias didácticas utilizadas por muchos docentes de todos los niveles (Sanjurjo, 2003, p. 36).

El concepto de objetividad -es decir, que el sujeto conoce las cosas como las cosas son- deriva de la idea de realidad única. Así como hay una única verdad, también hay una sola forma de conocer y una única forma de llegar a ella. Las personas sólo pueden reproducir fielmente la realidad, porque ella es independiente, inteligible de por sí y superior al hombre (Daros, 1983, pp. 89-91).

La creencia de que el conocimiento es algo dado, una entidad que existe de por sí, hace que sólo se la pueda ir descubriendo a través de los sentidos. Sin embargo, debido a su vastedad los hombres no 
pueden apropiarse de ella en su totalidad. Por el contrario, sólo podrá ser apreciada en lo particular. Para ser percibida, la realidad deberá ser parcelada en unidades discretas, dividida arbitrariamente en retazos de conocimiento. Pensar al conocimiento como entidad, como algo dado, indefectiblemente lleva a producir, enseñar y aprender conocimientos atomizados (Entel, 1988, p. 18). Por otro lado, los hombres y las mujeres aprenden estimulados por la realidad, que es exterior y superior a la persona. Las personas deben adaptarse a esa realidad inmutable y sólo pueden tener una actitud de pasividad ante los estímulos del medio (Daros, 1983, p. 91). En la situación de enseñanza dichos estímulos están representados por la exposición del docente 0 el texto de los libros. Considerar al conocimiento científico como un conocimiento rígido, inmutable y dogmático equivale a convertirlo en doctrina. Plantear estrategias didácticas desde un enfoque cientificista no es enseñar sino adoctrinar.

La otra postura, el constructivismo, sienta sus bases epistemológicas en la teoría filosófica denominada realismo crítico, que sostiene que la realidad está conformada por una tríada: el mundo natural (es decir, las cosas y las personas), el sujeto que conoce y la relación que se establece entre ambos. La realidad no es totalmente objetiva ni absolutamente subjetiva, es su relación en constante creación (Daros, 1983, pp. 94-95). Esta relación dialéctica constante permite afirmar que el conocimiento es el producto de un proceso dinámico y permanente de adaptación y de reconstrucción, un proceso siempre inacabado, que tiene una dimensión sensual, pero también intervienen en él factores culturales, socio-políticos y psicológicos (Sanjurjo, 2003, p. 37). Ignorar el proceso de construcción de un conocimiento implica desconocer los desafíos, contradicciones, pugnas, valores, marchas y contramarchas que permitieron su elaboración. En general, los docentes guiados por una concepción racionalista centran su preocupación en los contenidos sin tener en cuenta que el conocimiento es una totalidad compleja, atravesada por todos estos factores. "Considerar sólo los contenidos es, como todo error, una parcialidad arbitraria, muchas veces inconsciente, donde se toma a la parte por el todo" (Daros, 1983, p. 32).

\section{Las concepciones acerca del aprendizaje}

Como se ha expresado anteriormente, consciente o inconscientemente, cada decisión que un docente toma está fundada en la concepción que éste tiene respecto de qué es el aprendizaje y de cómo cada sujeto que aprende construye su conocimiento ${ }^{1}$ (Sanjurjo, 2014, p. 105).

Es posible enumerar una gran cantidad de teorías que tratan de explicar el proceso de aprendizaje ya que es un tema que preocupó y preocupa a la pedagogía, la psicología e incluso a la filosofía (Sanjurjo, 2003, p. 23). Se las puede clasificar de múltiples maneras. Sin embargo, a los fines de satisfacer el objetivo de este texto, se categorizarán como pertenecientes a dos grandes conjuntos según el enfoque epistemológico desde las cuales fueron elaboradas. El primer grupo está conformado por las teorías asociacionistas o conductistas y el segundo grupo se constituye con las teorías denominadas constructivistas, mediacionales o de la reestructuración.

Las teorías conductistas estudian el fenómeno del aprendizaje equiparándolo a un modelo de caja negra. Es decir, sólo se limitan a estudiar las relaciones entre los estímulos y las respuestas, sin tener en cuenta lo que ocurre en la mente del sujeto que aprende. Por el contrario, las teorías constructivistas consideran que cada persona que aprende es única. Tienen como uno de sus objetivos tratar de explicar lo que ocurre en la mente del que aprende, asumiendo que el aprendizaje es un proceso en el que el estudiante interactúa con el conocimiento, por lo cual se establecen interrelaciones entre ellos (Pérez Gómez, 1992b, pp. 36 - 37). Explican los procesos intelectuales valiéndose de un modelo teórico que hace referencia a una estructura o esquema que crea el sujeto en su mente, y que puede ser llamada estructura cognoscitiva.

Otra diferencia entre estas dos posturas está en la unidad básica de análisis de la que parten. Mientras los conductistas pueden ser considerados elementaristas al considerar que una totalidad puede descomponerse en sus partes, por ejemplo un concepto puede ser caracterizado a través de una simple lista de atributos, el enfoque cognitivo parte de unidades más molares, en las que el todo no es simplemente la suma de sus partes componentes.

Las estrategias didácticas que derivan del primer posicionamiento epistemológico estarán orientadas a generar los estímulos adecuados que garanticen el aprendizaje de los estudiantes. Esto significa que el profesor explica ordenadamente y en detalle todos los temas, en un ambiente silencioso, para que los alumnos puedan atender y recibir la información. El éxito de tal exposición se constata a partir de corroborar que las conductas observables de los alumnos se modifican en el sentido prefijado, es decir, a través de un examen en el que sólo se admitirán como correctas las respuestas que reproduzcan textualmente las explicaciones del profesor o lo expuesto en los libros de texto, que siempre son coincidentes.

Como contrapartida, las estrategias didácticas inspiradas en la postura constructivista tendrán el objetivo de crear las condiciones adecuadas para favorecer la interacción entre el nuevo conocimiento y la estructura cognoscitiva del sujeto. Con esta interacción se pretende que el conocimiento se incorpore a

\footnotetext{
${ }^{1}$ El conocimiento de las diversas teorías del aprendizaje por parte del docente no implica que éste deba aplicar los principios derivados de ellas directamente en el salón de clases. Sin embargo, sí le permiten hacer conscientes aquellos SBS, de forma tal de poder adoptar decisiones racionales y justificadas al planificar sus clases, en lugar de basarlas en "prescripciones tradicionales presentes en el folklore educativo y en los preceptos y ejemplos de sus propios maestros y colegas" (Ausubel, 1978, p. 20).
} 
dicha estructura determinando la modificación de ambos en un proceso de reestructuración y modificación que los involucra (Sanjurjo, 2003, p. 31). Estas estrategias tenderán a inducir al estudiante a tener una participación activa realizando tareas intelectuales o desarrollando destrezas físicas.

\section{Objetivo}

El objetivo fue reconocer y comprender algunos SBS sobre realidad, la ciencia, el conocimiento y el aprendizaje, en docentes que se desempeñan en el ciclo básico de la carrera de Medicina Veterinaria de la Facultad de Ciencias Veterinarias de la Universidad Nacional de Rosario (UNR).

\section{Materiales y métodos}

La siguiente indagación es parte de una tesis para optar por el título de Magister en Educación Universitaria, presentada en la Facultad de Humanidades y Artes de la UNR. En esta tesis se realizó un trabajo empírico por el cual se pudo establecer una relación entre los Supuestos Básicos Subyacentes de los profesores y el tipo de estrategias didácticas que utilizan los docentes según favorezcan o dificulten la integración de los conocimientos. Esta investigación cualitativa y transversal se realizó durante el año 2017 en la Facultad de Ciencias Veterinarias de la Universidad Nacional de Rosario. Para la recolección y tratamiento de datos, análisis y obtención de los resultados así como para elaboración de las conclusiones utilizamos una metodología de tipo etnográfica. Adoptar esta postura nos implicó un compromiso personal como investigadores ya que debimos interaccionar directamente con los sujetos que participaron de la investigación. Aceptamos y compartimos la idea de considerar al investigador como un sujeto reflexivo (Ameigueiras, 2006, pp. 107-148).

Se realizaron entrevistas en profundidad a los profesores a cargo de las cátedras que integran el ciclo básico de la carrera Medicina Veterinaria:

- Anatomía Descriptiva y Comparada I

- Histología I y Embriología Básica

- Anatomía Descriptiva y Comparada II

- Histología Il y Embriología Especial

- Fisiología

En esta investigación realizamos entrevistas abiertas para las que utilizamos una guía general basada en ejes temáticos, seleccionados con el objetivo de generar y orientar un intercambio reflexivo. Este tipo de entrevistas nos permitió manejar con toda flexibilidad el ritmo, la estructura, las preguntas y las repreguntas. En ellas, los entrevistados tuvieron toda la libertad para expresar sus ideas y puntos de vista.

Cada entrevistado fue informado sobre el carácter voluntario, el contexto investigativo de la aplicación, el destino y la confidencialidad de los datos. Para desarrollar la tarea analítica utilizamos el programa para tratamiento de datos cualitativos Atlas $\mathrm{Ti} 8.4 \AA$ que se basa en el método Teoría Fundamentada propuesto por Strauss y Corbin (1998).

\section{Resultados y discusión}

Según la metáfora que utiliza Alvin Goudner (1970), "Ios SBS proveen el capital intelectual heredado que recibe el teórico mucho antes de llegar a serlo, y que luego invierte en sus roles intelectuales y científicos, fundiéndolos con su preparación técnica” (Alvin Goudner, 1970, p. XX). Esta afirmación abre nuestra discusión sobre los resultados obtenidos, a través de la cual hemos corroborado en términos generales que el pensamiento de muchos profesores universitarios estaría condicionado por la cultura occidental, capitalista y positivista en que se formaron.

\section{Las concepciones acerca de la realidad, la ciencia y el conocimiento}

Concebir la realidad como algo estático, preexistente, con leyes inmutables que el investigador debe descubrir, es propio de las personas que se desarrollaron bajo el influjo de la cultura positivista. Esta concepción de la realidad no solo impregna la forma de pensar de la persona común, se hace presente también en la mentalidad de la gran mayoría de los profesionales, especialmente aquellos formados en las llamadas "ciencias duras".

Existe también otra forma de pensar la realidad que conduce a concebirla no como algo preexistente sino como una construcción social y cultural propia de cada sujeto y/o comunidad. Estas dos posturas pueden ser consideradas como los extremos de un continuo complejo dentro del cual se puede ubicar la forma de pensar de cada persona. Efectivamente, lo más probable es que si analizamos la manera en que cada sujeto considera la realidad nos encontremos con que pocos o ninguno piensa exactamente de una $u$ otra manera, sino que algunos tienen una manera de pensar más cercana a la concepción positivista y otros una posición más cercana a la concepción constructivista.

Al analizar lo manifestado por uno de los docentes entrevistados (D1) nos encontramos con expresiones como:

"o sea que son piezas que se preparan y se usan también para demostraciones, no para que trabajen los alumnos, no hay disección"

"mayor cantidad de piezas"

"material que podría ser valioso" 
REVISTA DE LA ESCUELA DE CIENCIAS DE LA EDUCACIÓN, AÑO 16, NRO. 15, VOL. 2, JULIO A DICIEMRE DE 2020. PÁGINAS 58-67. ISSN 2362-3349 (EN LÍNEA). LOS SUPUESTOS BÁSICOS SUBYACENTES DE LOS DOCENTES DE CIENCIAS VETERINARIAS DE LA UNIVERSIDAD NACIONAL DE ROSARIO. RODOLFO DARÍO GARCÍA. GRISELDA MUÑOZ.

"conseguir más material para trabajo de disección"

"que ellos vayan construyendo el conocimiento desde la propia realidad"

"tener un lugar adecuado cómo para que los equipos puedan trabajar en laboratorio sería algo muy interesante."

A partir de un primer análisis, podemos destacar la sobrevaloración que el docente hace de las piezas del museo como recurso didáctico. Sin embargo, es el propio docente el que afirma que estos elementos son para "demostraciones". Indica, de esta forma, que no se utilizan para que los estudiantes trabajen sobre estos elementos para re-construir lo que estudiaron en las clases de teoría, por el contrario, se espera que refuercen lo desarrollado en la teoría.

Una posible interpretación es que el profesor considera la realidad como algo estático. Si esto fuera así, es posible que el docente conciba a la disección como la instancia en la que los alumnos corroboran el conocimiento que se encuentra en la bibliografía. Podemos relacionar esta manera de concebir la enseñanza de la anatomía con la vieja concepción empirista "que reducía el conocer a una mera impresión sobre el sujeto de los datos del mundo externo" (Linaza Iglesias, 2002, p. 109).

En general, las expresiones del profesor tienden a dar forma al concepto, propio del pensamiento positivista, de que la observación de las piezas de museo es la mejor forma de aprender esta disciplina. Esta manera de entender la enseñanza se puede relacionar con uno de los principios fundamentales de la escuela conductista, la ley del reforzamiento. Este principio fue desarrollado por Burrhus Skinner en su sistema del condicionamiento operante (Chaplin, 1978, pp. 203-207). Esta idea, pondría en evidencia que los docentes que avalan esta postura estiman que lo más conveniente para un buen aprendizaje es que los estudiantes puedan ratificar en muchas piezas el conocimiento que les fue entregado en la teoría. En otro fragmento de su discurso expresa, en lugar de leer lo que otros hicieron, sino ver ellos por sí mismos, y a su vez leyendo la bibliografía y discutiendo con lo que dicen los autores y sacando una conclusión.

Esta afirmación podría ratificar que el docente tiene una concepción de la realidad como algo único, que la descubrieron otros, que la escribieron en libros y que él debe entregar a sus alumnos ese conocimiento que considera como algo real. Este pensamiento correspondería a lo que Tadeu da Silva (1998) denomina una "epistemología realista, [en la que] el conocimiento se concibe simplemente como reflejo de una realidad que está ahí, a la que se puede acceder directamente" (Tadeu da Silva, 1998, p. 65). A través de este recurso, los docentes prueban a los estudiantes que el conocimiento que ellos les transmitieron en las clases teóricas es verdadero.

De la misma manera, el docente 5 sostuvo: "Igualmente, sigo pensando que la mejor forma de dar anatomía es con los preparados anatómicos, en la sala de anatomía y que el alumno vea y palpe."

Este comentario nos remitiría al antiguo método intuitivo para la enseñanza que se sustenta en la didáctica sensual empirista, propia del paradigma positivista del siglo XIX. "Su característica es ofrecer, en lo posible, elementos sensibles a la percepción y a la observación de los alumnos. Es la didáctica de las lecciones de las cosas, también llamada a veces didáctica de la tiza de color" (Aebli, 1958, pp. 14-15). Una de las posibles interpretaciones es que para estos profesores la realidad existe per se y que la manera de acceder a ella es a través de los sentidos. El profesor, según este paradigma, cumple con su función al mostrarles a los alumnos cómo es la realidad haciendo que vea y palpe las diferentes estructuras anatómicas.

Otro docente (D3), hablando de las interrelaciones entre las cátedras, expresó:

Construirían conocimiento a partir de una base que sea acordada por todas las cátedras. Entonces, si vos tenés una base que coincide, sobre esa base seguís construyendo. En cambio, si cada uno le dice una cosa distinta sobre el mismo tema. Ahí es como que los confundís, y finalmente el resultado no es el que vos querés.

Consideramos que este comentario es un indicador de cómo el paradigma positivista puede estructurar la manera de pensar en los profesores. Esta cita anuncia la idea de que los alumnos aprenden mejor si se les presenta el conocimiento pre elaborado y en una única versión. Según la postura positivista, a los alumnos debe llegar un discurso que ignore

Las dimensiones conflictivas de la realidad y, por lo tanto, de la cultura. Se pretende propagar entre el conjunto de los estudiantes una concepción del conocimiento y de la experiencia humana estática, libre de valores, una teoría del consenso de la ciencia (Apple, M. 1986, citado por Torres Santomé, 1994, p. 110).

Esta manera de pensar se sustenta en la idea de que hay una única forma de conocer y una verdad que debe ser aprendida.

Por otro lado, el docente habla de obtener un resultado que espera sea de una determinada manera. Esto indicaría que los docentes que piensan de esta manera conciben a la realidad como algo fijo, que puede ser usada como modelo con el cual comparar los aprendizajes. Esto explicaría por qué los profesores que se formaron dentro del paradigma positivista sostienen que, para valorar lo que los 
REVISTA DE LA ESCUELA DE CIENCIAS DE LA EDUCACIÓN, AÑO 16, NRO. 15, VOL. 2, JULIO A DICIEMRE DE 2020. PÁGINAS 58-67. ISSN 2362-3349 (EN LÍNEA). LOS SUPUESTOS BÁSICOS SUBYACENTES DE LOS DOCENTES DE CIENCIAS VETERINARIAS DE LA UNIVERSIDAD NACIONAL DE ROSARIO. RODOLFO DARÍO GARCíA. GRISELDA MUÑOZ.

estudiantes aprendieron, se debe comparar lo que los alumnos expresan en los exámenes con esa versión única de la realidad.

Al igual que lo que sucede con otras expresiones, también está presente la idea de que los profesores son los encargados de decir cómo es la realidad, que ellos son los que saben, lo cual se puede traducir como un mensaje autoritario.

Cuando interrogamos sobre la relación entre la investigación y la enseñanza, D4 afirmó:

Muchos de los temas que se abordaron en la investigación, en [mi] tesis, después se aplicaron en el dictado de los cursos regulares. Antes no existían en el programa analítico de la histología contenidos que tuvieran que ver con la histología de los peces y a partir de ahí [de lo que hice en mi tesis doctoral] muchos de los conocimientos desarrollados durante [mi] tesis fueron volcados a las clases de grado, porque es una forma de retro alimentación ${ }^{2}$.

En este caso el profesor utilizaría a la investigación como parte del proceso de enseñanza solamente para poder aumentar los contenidos de la materia y no para mostrar a los alumnos la relatividad de los conocimientos. Según una concepción que podríamos denominar Flexneriana ${ }^{3}$ de la enseñanza de la ciencia, esta debe comenzar con el estudio de la disciplinas básicas pertinentes y debe seguir con el aprendizaje de sus aplicaciones. No se contempla la posibilidad de incluir la investigación. Por el contrario, se asume como natural que la investigación y la enseñanza se desarrollen en tiempos y lugares separados. Muchos docentes conciben el conocimiento profesional como

Información privilegiada o como competencia. Conciben a la enseñanza como transferencia de la información; el aprendizaje como recepción de lo dicho y de asimilación de la información. El saber qué tiende a ser prioritario sobre el saber cómo; y el saber cómo, cuando hace su aparición, adopta un planteamiento tecnológico (Schön, 1992, p. 270).

En general, los docentes tienden a presentar los conocimientos científicos como verdaderos, como algo que ya se sabe cómo es. Tienden a no mostrar desconocimientos o dudas y si enseñan o comentan avances científicos lo hacen de manera que se tiene la impresión de que se ha descubierto finalmente la verdad. Este supuesto, de que el docente conoce, o dicho de otra manera, que no desconoce, se deriva de la concepción positivista del docente como alguien poderoso y superior. Por el contrario Edgard Morin afirma:

La educación debe mostrar que no hay conocimiento que no esté, en algún grado, amenazado por el error y por la ilusión. [...] Un conocimiento no es un espejo de las cosas o del mundo exterior. Todas las percepciones son a la vez traducciones y reconstrucciones cerebrales (Morin, 1999b, p. 5)

Luego, el mismo docente comenta:

Las clases teóricas, que son más de tipo clase magistral se dictan una vez a la semana, están todas las comisiones juntas. Después tenemos clases teórico-prácticas, dónde generalmente en esas clases hay una introducción teórica bastante importante y después hay una actividad práctica que puede llegar a ser la visualización de preparados con el microscopio. Después tenemos los prácticos que son similares a lo anterior, nada más que no hay dictado de contenido teórico, salvo, a lo mejor, una breve introducción recordando algunos contenidos, es estrictamente práctica. Normalmente esas clases..., digamos, tuvieron una clase teórica específica para desarrollar el tema.

Aquí surge la idea de que los conocimientos se enseñan en la clase teórica y que en otra clase, la clase práctica, completamente separada, los alumnos confirman la realidad en los preparados con el microscopio. Esta postura de separación de lo teórico de lo práctico lleva a que los alumnos aprendan los conceptos descontextualizados, aislados de su aplicación práctica. Uno de los objetivos que debemos

\footnotetext{
${ }^{2}$ Los agregados o cambios que hice, para una mejor comprensión de la cita, están entre corchetes.

${ }^{3}$ El adjetivo flexneriano hace referencia a Abraham Flexner (1866 - 1959), autor del denominado informe Flexner en 1910.

Se suelen denominar de esta manera a aquellos planes de estudios médicos en los cuales existe una clara división entre un período o ciclo inicial de disciplinas básicas, seguido por otro dedicado a los estudios clínicos. De hecho, esta propuesta -una entre las varias recomendaciones realizadas por este autor- ha devenido en la más conocida, cuando no la única, que se identifica con su nombre. Llama la atención que siendo tan criticada haya resistido casi 100 años de aplicación y actualmente se encuentre vigente en la mayor parte de las escuelas de medicina del mundo (Vicedo Tomey, 2002, s/p).
} 
perseguir los docentes, especialmente en el ámbito universitario, es que los estudiantes logren que los conceptos que conforman las teorías de las distintas disciplinas, y que sirven para un análisis más riguroso de la realidad, se incorporen al pensamiento del aprendiz como poderosos instrumentos y herramientas de conocimiento, que permitan la resolución de problemas y no como meros adornos retóricos que se utilizan para aprobar los exámenes y olvidarlos después. De esta manera, sería esperable que el aprendizaje de los conceptos se produzca en forma similar a como tiene lugar el aprendizaje de las herramientas requeridas para la realización de cualquier oficio. Sin embargo, como en estos casos, los alumnos universitarios muchas veces toman contacto con los conceptos abstractos de las disciplinas de modo sustancialmente teórico, al margen del contexto de la práctica profesional, de la comunidad y de la cultura donde aquellos conceptos adquieren sentido funcional, como herramientas útiles para hacer una interpretación crítica de la realidad y sobre todo como herramientas de intervención (Pérez Gómez, 1992c, p. 109-110).

A continuación se presenta un párrafo extraído de la entrevista al docente D5 que nos conduce a reflexionar sobre la persistente convicción en los docentes de concebir la teoría como algo que precede la práctica, e incluso con la que no necesariamente guarda dependencia.

Tomamos muchísimos cursos de formación de Anatomía: con Barone, con König, con Lahunta. Él decía [El docente X4], y no me olvido de estas palabras: "el profesor de anatomía que da el teórico tiene que bajar a los trabajos prácticos para estar con su alumno y mostrarle la contundencia de los actos. Lo que se dice en los teóricos tiene que estar reflejado en lo que ellos ven en los prácticos". Bueno, y eso es una gran realidad que siempre me quedó y mientras lo pude hacer, lo hice.

Sin embargo, en contraposición a la opinión del profesor, es posible pensar la enseñanza desde una perspectiva más integradora. Se trataría de amalgamar lo conceptual con lo técnico. Esto posibilitaría que los docentes ofrezcan una perspectiva más completa, les permitiría ampliar el sentido restringido de lo técnico,

Porque ninguna técnica es neutral para quien la recibe, para quien la práctica o para el mensaje que transmite. Y porque, en nuestro caso, el saber hacer, el cómo, encuentra sus justificaciones en criterios que van más allá de la racionalidad instrumental, más allá del logro de la eficacia de los medios, debiendo encontrar justificación en relación a los fines a los que sirve (Gimeno Sacristán, 1992).

Como en los casos anteriores, los comentarios de estos docentes nos ubican frente a SBS mayormente vinculados a la perspectiva positivista desde la cual se concibe al mundo como algo estático, al que se accede a partir de experiencias sensoriales. Además, y probablemente sea uno de los SBS más importantes, los docentes reconocerían a la ciencia, al conocimiento, como libre de valores.

\section{Las concepciones acerca del aprendizaje}

De acuerdo a lo que presentamos en la introducción teórica, las posturas más cercanas a la concepción conductista de la enseñanza se basan, entre otros, en el supuesto que dice que los estudiantes deben recibir el conocimiento, que éste debe haber sido elaborado, procesado y recortado por el docente. Sin embargo, cómo bien lo expresa Mario Carretero (1993):

Una idea con la que posiblemente están de acuerdo muchos psicólogos en la actualidad es que el aprendizaje es un proceso constructivo interno. [...] no basta la presentación de la información a un individuo para que la aprenda, sino que es necesario que la construya mediante su propia experiencia interna. El profesor debería tener este principio muy presente porque la visión tradicional y más extendida de la enseñanza se basa en la idea de que la transmisión de conocimientos es del profesor al alumno. Es decir, el primero va depositando información en la mente del alumno y este la va almacenando de manera más o menos ordenada. En la actualidad son muchos los datos que hablan en contra de esta concepción (Carretero, 1993, p. 57).

Los profesores entrevistados, en general, piensan que sus alumnos no pueden comprender un tema complejo si no se los enseña, si no se los explica previamente. Un ejemplo de esta forma de pensamiento la podemos encontrar en las afirmaciones que hace el docente 5:

D5- "Estoy elaborando una estrategia que ya está puesta en marcha en algunas facultades. Por ejemplo: el acortamiento de la carga horaria, ya muchas de las cátedras de anatomía están en ochenta horas y han bajado, porque han empezado a depurar contenidos de una manera bastante interesante, el caso de Esperanza y de Pico; y lo otro es que ya algunos han dejado de dar los teóricos [...] me cuesta lo de los teóricos, pero... si les está dando resultados a ellos"

E- ¿Por qué le cuesta? 
REVISTA DE LA ESCUELA DE CIENCIAS DE LA EDUCACIÓN, AÑO 16, NRO. 15, VOL. 2, JULIO A DICIEMRE DE 2020. PÁGINAS 58-67. ISSN 2362-3349 (EN LÍNEA). LOS SUPUESTOS BÁSICOS SUBYACENTES DE LOS DOCENTES DE CIENCIAS VETERINARIAS DE LA UNIVERSIDAD NACIONAL DE ROSARIO. RODOLFO DARÍO GARCíA. GRISELDA MUÑOZ.

D5- "Me cuesta porque me parece que es una situación que el alumno la necesita".

\section{E- ¿Por qué?}

D5- "Y, porque anatomía es una materia muy complicada que requiere de una explicación. Por supuesto que una cosa es la anatomía... o el teórico sin la ayuda de lo que son las imágenes y la otra es con la ayuda de las imágenes. Si vos le ponés un muy buen power point o lo ayudás... a mí me gusta mucho esquematizar en el pizarrón"

Los conceptos que presenta el docente contrastan con el carácter productivo que debe tener el aprendizaje del conocimiento científico. En contraposición a la opinión del profesor, consideramos que la ausencia de actividad por parte del alumno, entendida también como la posibilidad de interpretar o resolver situaciones a partir de conceptos elaborados por otros, cosifica la información. El carácter dinámico del conocimiento es el que determina la utilización de una serie de estrategias didácticas que incluyen la exigencia del trabajo del estudiante sobre la información y no su mera recepción y reproducción.

Al contrario de lo que concibe el profesor como una buena enseñanza de la anatomía, coincidimos con Alfredo Furlán (1989) en que:

El contenido científico no puede ser transmitido como conceptos teológicamente garantizados por la autoridad del profesor o por las sagradas escrituras de los textos; este enfoque es pura anticiencia pues no ayuda a los estudiantes a comprender el conocimiento científico como un cuerpo en construcción continua, históricamente condicionado, que ofrece mecanismos abiertos para su permanente actualización o para su reestructuración; genera solamente una actitud de pedantería ilustrada, basada en la creencia de que el recuerdo de una masa de información es suficiente para transitar el camino hacia un status científico. Si se pretende que se aprenda un contenido científico hay que buscar las formas idóneas que implican concebir al estudiante como un productor potencial y no como un repetidor. La ciencia exige el combate cotidiano de los maestros en contra de las esclerosis cerebrales tempranas de los estudiantes $-\mathrm{y}$ de ellos mismos- producidas por los hábitos memorísticos y por las cegueras sobre sectores de la realidad que quedan fuera del recorte disciplinario del objeto de estudio (Furlán, 1989, p. 63).

Más adelante, el mismo docente, analizando los resultados de cambio curricular que se dio en la Facultad de Ciencias Médicas de la UNR, afirmó:

D5- "esto se lo decíamos a la decana de medicina cuando hizo el plan de estudio: el cambio del plan de estudio está bárbaro, las UABP están bárbaras, pero si usted no les cambia la cabeza a los docentes que les tienen que marcar el rumbo a los chicos no sirve de nada el cambio del plan de estudio. Porque no tenía recursos humanos adaptados a ese plan de estudios. Y fue realmente un fracaso eso. Una cosa traída de los pelos que han tenido que hacer un montón de prerrogativas para que los chicos puedan seguir avanzando. [...] están dando cursadas de anatomía enteras, porque los chicos no daban la cursada de anatomía entera, entonces, cuando iban al módulo fulanito y les tocaba como líder una antropóloga, la antropóloga no tenía ni idea de lo que era la aorta o la vena cava, ni por dónde venía".

Se podría interpretar, siguiendo la tendencia que vienen marcando las citas anteriores, que el supuesto que guía el discurso y los pensamientos del profesor se sustentan en la idea de que los estudiantes solo pueden aprender si el docente les indica lo que deben estudiar. Según sus propias palabras, los profesores son los encargados de "marcar el rumbo a los chicos". El pensamiento de muchos profesores se corresponde con una concepción altamente conductista de la enseñanza y contrasta totalmente con lo que se considera, desde la perspectiva constructivista la función del profesor y de las instituciones educativas: "Más que transmitir información, [...] debe orientarse a provocar la organización racional de la información fragmentaria recibida" (Pérez Gómez, 1992a, p. 28).

Las reflexiones del profesor, representan la manera de pensar de muchos de nosotros que esperamos que nuestros estudiantes incorporen ciertos contenidos de manera acrítica, por el solo hecho de que se los damos. No sólo eso, además esperamos que los repitan textualmente en el examen.

Por otro lado, en la última parte del extracto el docente comentó que los estudiantes no aprendieron satisfactoriamente los contenidos específicos de Anatomía e indicó que esta es la razón por la que se repitieron las cursadas de anatomía, pero con un estilo más tradicional. Según su punto de vista, como el líder del módulo no es un docente del área de anatomía, carece de los conocimientos disciplinares y por ello los estudiantes no pudieron aprender anatomía. Es decir, él piensa que los alumnos necesitan al docente para que les enseñe lo que solos no pueden aprender.

Se podría inferir que el profesor, desde su perspectiva conductista de la enseñanza, considera que una buena clase es aquella en la que el docente se ciñe a enseñar los contenidos disciplinares prescriptos por el programa de la materia. Esta es la forma clásica en que se entiende la función del profesor. Los docentes que adhieren a esta manera de concebir la clase, tal vez sin saberlo, reproducen 
la cultura positivista en la que ellos mismos se formaron. Estas enseñanzas implícitas comprenden, entre muchas otras, la idea de la ciencia como algo acabado, la idea de la relación asimétrica del poder y la consecuente pérdida del sentido democrático de la convivencia, promueve el trabajo individual, la falta de cooperación y la competitividad, llevando todo esto a los alumnos a un estado de aislamiento e indefensión. Por otra parte, si consideramos que el sentido y el significado no se construyen en forma aislada ni en el vacío, es este estado de segregación el que le dificulta a los estudiantes otorgarle significados a los conceptos que el profesor explica. Por el contrario, favorece la memorización y el aprendizaje compartimentalizado (Tadeu de Silva, 1998, pp. 59-75).

Al contrario de lo que opina el profesor, una enseñanza más democrática, que tienda a favorecer la integración de los conocimientos, se debe sustentar en que la función del docente no es la de presentar la información, sino que es la de guiar a los estudiantes en la búsqueda, selección e interpretación de los contenidos, para que luego ellos mismos los resignifiquen y construyan su propio discurso. En este caso, la función del maestro, suele ser ayudar a los estudiantes para que puedan defender su construcción cognitiva con argumentos sólidos, antes que meterles a la fuerza grandes cantidades de información, al viejo estilo enciclopedista. Aplicando estos conceptos al ejemplo que cita el profesor, tal vez, un docente con una buena formación humanística sea, incluso, más adecuado para guiar a los alumnos que lo que él consideraría un buen profesor de anatomía.

Muchos profesores suponen que el estudiante incorpora la información de manera lineal, por lo que sostienen que hay que presentarles los temas en forma ordenada. De esta manera, es coherente que sostengan que la asignatura solo puede ser enseñada por un docente con sólida formación disciplinar. Los docentes que se formaron con esta idea, propia de la pedagogía conductista, no pueden comprender que el conocimiento se construye y se remodela constantemente. Piensan que el alumno incorpora el conocimiento y este queda fijo y estático en su mente. De allí su creencia de que el conocimiento al que acceden los estudiantes debe estar bien estructurado. Piensan que eso que aprenden es lo que sabrán siempre. No comprenden que el conocimiento adquirido en la primera lectura de un tema será reformado innumerable cantidad de veces a lo largo de su vida como estudiantes y como profesionales.

Una concepción un poco más cercana al extremo constructivista la encontramos en los dichos del docente 2

D2- "Bien, hace dos años dijimos: nosotros pusimos, o en el plan de estudios dice ciento cincuenta horas, que son ciento cincuenta horas que el docente cree las necesarias para impartir la asignatura [...] nosotros decimos que es al revés, nosotros decimos que son ciento cincuenta horas para que el alumno aprenda, no para que el docente dicte, que son dos cosas totalmente diferente. Entonces, la temática de juego que nosotros tenemos ahora es que el alumno, las diez horas semanales que tiene de histología las utilice para aprender, para estudiar. Invertimos el rol. Entonces, hay seminarios y prácticos. Los seminarios y los prácticos se evalúan. Para eso tiene una introducción de quince minutos, unas consignas básicas que son lo mínimo indispensable que nosotros creemos que el alumno debe saber acerca de tal o cual tema. Eso es lo que nosotros exigimos. Bien, para eso tiene unas consignas a contestar en una hora o en una hora y cuarto, con la bibliografía. Pero no apunte, no, la bibliografía. O sea, libros. Entonces, de esa manera, dos cosas, primero aprende a manejar libros, punto número uno, aprende a hacer las síntesis o los resúmenes, tercero, aprende a interpretar una consigna y a contestar la consigna, cuarto, una vez que termina la clase, pasan, de acuerdo a la cantidad de grupos, un relator por grupo que va a contestar las consignas que se le dieron, para debate entre los cinco relatores y la clase, si están todos de acuerdo, si encontraron lo mismo o no"

Las palabras de este profesor indicarían que entre los SBS que guían sus decisiones está la idea de que los estudiantes pueden tener un desempeño más autónomo. La decisión de reemplazar los teóricos por una estrategia didáctica en la que los estudiantes tengan más protagonismo la pueden llevar adelante los docentes que consideran al alumno capaz de elaborar su propio conocimiento. Si bien no estamos ante una propuesta de enseñanza totalmente liberal, en contraposición al ejemplo anterior, vemos que en este caso se estimula, al menos en las intenciones, un debate, una discusión entre pares. Este tipo de estrategias permiten que los estudiantes se comprometan más con sus pensamientos y, de esta manera, ponen en juego una serie de sentimientos, todo lo cual, como es sabido, redunda en una mejora del aprendizaje.

\section{Conclusiones}

Los SBS se van conformando en el sujeto a lo largo de la vida, tanto por su interacción social cotidiana como por las influencias que ejercen las distintas instancias educativas por las que esa persona va transcurriendo. En el caso de los docentes del ciclo básico de la Facultad de Ciencias Veterinarias, encontramos que estos SBS Se estructurarían en torno a ideas y conceptos más bien cercanos al paradigma positivista.

En general, hallamos que los docentes conciben la realidad como algo que existe más allá del sujeto cognoscente y no como una construcción social. Este supuesto, que es uno de los pilares del pensamiento positivista, se convierte en el sustento epistemológico de otros que son los que terminan de conformar las concepciones de estos docentes. 
REVISTA DE LA ESCUELA DE CIENCIAS DE LA EDUCACIÓN, AÑO 16, NRO. 15, VOL. 2, JULIO A DICIEMRE DE 2020. PÁGINAS 58-67. ISSN 2362-3349 (EN LÍNEA). LOS SUPUESTOS BÁSICOS SUBYACENTES DE LOS DOCENTES DE CIENCIAS VETERINARIAS DE LA UNIVERSIDAD NACIONAL DE ROSARIO. RODOLFO DARÍO GARCía. GRISELDA MUÑOZ.

Si bien las conclusiones precedentes podrían ser aplicadas al conjunto de los docentes, es importante remarcar la existencia de otros hallazgos que sugieren la existencia de otras concepciones más cercanas a los SBS originados en el paradigma constructivista, aunque claramente debilitadas 0 marginadas por el imperio de la perspectiva positivista dominante. Algunos de los docentes manifestaron que la responsabilidad de aprender es de los alumnos y expresaron su convencimiento de que los alumnos deberían tener un desempeño más autónomo.

\section{Referencias bibliográficas}

Aebli, H. (1958). Una didáctica fundada en la psicología de Jean Piaget. Buenos Aires. Argentina: Kapeluz.

Ameigueiras, A. R. (2006). El abordaje etnográfico en la investigación social. En: I., Vasilachis de Gialdino, (coord.) (2006). Estrategias de investigación cualitativa. Colección Biblioteca de Educación. Herramientas Universitarias. Barcelona. España: Editorial Gedisa.

Ausubel, D.; Novack, J. y Hanesian, H. (1978). Psicología educativa. Un punto de vista cognoscitivo. Mexico: Trillas.

Carretero, M. (1993). Constructivismo y educación. Buenos Aires. Argentina: Aique.

Chaplin, J. P.; Krawiec y Theophile S. (1978). Psicología: Teorías y sistemas. México: Interamericana.

Daros, W. R. (1983). Epistemología y didáctica. Rosario. Argentina: Ediciones Matética.

Entel, A. (1988). Escuela y conocimiento. Buenos Aires. Argentina: Miño y Dávila - FLACSO.

Furlán, A. J. (1989). Metodologías de la enseñanza. En: Furlán, A. y otros (1989). Aportaciones a la didáctica de la educación superior. México: UNAM.

Gimeno Sacristán, J. (1992). El currículum: ¿Los contenidos de la enseñanza o un análisis de la práctica? En: J. Gimeno Sacristán y A. Pérez Gómez (1992). Comprender y transformar la enseñanza. Madrid. España: Ediciones Morata.

Gouldner, A. W. (1970). La crisis de la sociología occidental. Traducción: Miguez, Nestor. Buenos Aires Argentina: Amorrortu.

Linaza Iglesias, J. L. (2002). Cambios en la concepción de la educación. En: F. J. Laporta, (Ed.) (2002). La enseñanza del derecho. Universidad Autónoma de Madrid. Madrid. España: Anuario de la Facultad de Derecho de la Universidad Autónoma de Madrid.

Morin, E. (1999b). Los siete saberes necesarios para la educación del futuro. Traducción: Vallejo Gómez, Mercedez. París. Francia: Editorial Santillana - UNESCO.

Pérez Gómez, Á. (1992a). Las funciones sociales de la escuela: de la reproducción a la reconstrucción crítica del conocimiento y la experiencia. En: J., Gimeno Sacristán; A., Pérez Gómez (1992). Comprender y transformar la enseñanza. Madrid. España: Ediciones Morata.

Pérez Gómez, Á. (1992b). Los procesos de enseñanza-aprendizaje: análisis didáctico de las principales teorías del aprendizaje. En: J., Gimeno Sacristán y A., Pérez Gómez (1992). Comprender y transformar la enseñanza. Madrid. España: Ediciones Morata.

Pérez Gómez, Á. (1992c). Enseñanza para la comprensión. En: J., Gimeno Sacristán y A., Pérez Gómez (1992). Comprender y transformar la enseñanza. Madrid. España: Ediciones Morata.

Sanjurjo, L. O. y Vera, M. T. (2003). Aprendizaje significativo y enseñanza en los niveles medio y superior. Rosario. Argentina: Homo Sapiens.

Sanjurjo, L. O. y Trillo Alonso, F. (2014). Didáctica para profesores de a pie: propuestas para comprender y mejorar la práctica. Rosario. Argentina: Homo Sapiens Ediciones.

Schön, D. (1992). La formación de profesionales reflexivos. Hacia un nuevo diseño de la enseñanza y el aprendizaje en las profesiones. Barcelona: Paidós.

Strauss, A. y Corbin, J. (1998). Bases de la investigación cualitativa. Técnicas y procedimientos para desarrollar la teoría fundamentada. Traducción: Eva Zimmerman. Colombia: Editorial Universidad de Antioquia.

Tadeu da Silva, T. (1998). Cultura y currículum como prácticas de significación. En: Revista de Estudios del Currículum, (1)1. ISNN $1138-5669$.

Torres Santomé, J. (1994). Globalización e interdisciplinariedad: el curriculum integrado. Madrid. España: Ediciones Morata.

Vicedo Tomey, A. (2002). Abraham Flexner, pionero de la educación médica. En: Revista Cubana de Educación Médica Superior. (16)2. Disponible en: http://scielo.sld.cu/pdf/ems/v16n2/ems10202.pdf (Consultado: 05/02/2019) 